This manuscript has been accepted for publication in Accident Analysis and Prevention. The manuscript will undergo copyediting, typesetting, and review of the resulting proof before it is published in its final form. Please note that during the production process errors may be discovered which could affect the content, and all disclaimers that apply to the journal apply to this manuscript. A definitive version was subsequently published in Buendia R, Candefjord S, Fagerlind H, Bálint A and Sjöqvist BA. On scene injury severity prediction (OSISP) algorithm for car occupants. Accident Analysis and Prevention, vol 81, pp. 211-217, August 2015, DOI 10.1016/j.aap.2015.04.032 


\title{
On Scene Injury Severity Prediction (OSISP) Algorithm for Car Occupants
}

\author{
Ruben Buendia ${ }^{\mathrm{a}, \mathrm{b}, \mathrm{c}, 1}$, Stefan Candefjord ${ }^{\mathrm{a}, \mathrm{b}, \mathrm{c}, 1, *}$, Helen Fagerlind ${ }^{\mathrm{d}, \mathrm{b}}$, András Bálint ${ }^{\mathrm{d}, \mathrm{b}}$, Bengt Arne \\ Sjöqvist ${ }^{a, b, c}$ \\ ${ }^{a}$ Department of Signals and Systems \\ Chalmers University of Technology \\ 412 96, Gothenburg, SWEDEN \\ ${ }^{b}$ SAFER Vehicle and Traffic Safety Centre at Chalmers, SWEDEN \\ ${ }^{c}$ MedTech West \\ Sahlgrenska University Hospital \\ Röda Stråket 10 B \\ 41345 Gothenburg, SWEDEN \\ ${ }^{d}$ Department of Applied Mechanics \\ Chalmers University of Technology \\ 412 96, Gothenburg, SWEDEN
}

\begin{abstract}
Many victims in traffic accidents do not receive optimal care due to the fact that the severity of their injuries is not realized early on. Triage protocols are based on physiological and anatomical criteria and subsequently on mechanisms of injury in order to reduce undertriage. In this study the value of accident characteristics for field triage is evaluated by developing an On Scene Injury Severity Prediction (OSISP) algorithm using only accident characteristics that are feasible to assess at the scene of accident. A multivariate logistic regression model is constructed to assess the probability of a car occupant being severely injured following a crash, based on the Swedish Traffic Accident Data Acquisition (STRADA) database. Accidents involving adult occupants for calendar years 2003-2013 included in both police and hospital records, with no missing data for any of the model variables, were included. The total number of subjects was 29 128, who were involved in 22607 accidents. Partition between severe and non-severe injury was done using the Injury Severity Score (ISS) with two thresholds: ISS > 8 and ISS $>15$. The model variables are: belt use, airbag deployment, posted speed limit, type of accident, location of accident, elderly occupant ( $>55$ years old), sex and occupant seat position. The area under the receiver operator characteristic curve (AUC) is 0.78 and 0.83 for ISS $>8$ and ISS $>15$, respectively, as estimated by 10-fold cross-validation. Belt use is the strongest predictor followed by type of accident. Speed limit, age and accident location contribute substantially to increase model accuracy, whereas sex and airbag deployment contribute to a smaller extent and seat position is of limited value. These findings can be used to refine triage protocols used in Sweden and possibly other countries with similar traffic environments.
\end{abstract}

Keywords: triage, prehospital care, logistic regression, postcrash, traffic safety.

\section{Introduction}

The Swedish parliament enforced "Vision Zero" in 1997 stating the long-term goal that no loss of life or severe injuries shall occur due to road traffic accidents. Although the mortality has been successfully

*Corresponding author at: Signals and Systems, Chalmers University of Technology, 412 96, Gothenburg, SWEDEN. Phone: +46 733821537

Email address: stefan.candefjord@chalmers.se (Stefan Candefjord)

${ }^{1}$ These authors contributed equally to this work. They are co-first author. 
decreased there are still around 300 people killed and 3000 severely injured yearly (Saxton, 2013). Much effort has been devoted to improving crashworthiness and developing active safety of vehicles with good results; however, improving the prehospital care process has received less attention, it is also fundamental for decreasing mortality and mitigate injury (Murad et al., 2012; MacKenzie et al., 2006).

Minimizing the delay to definitive treatment has been shown to decrease mortality substantially (Haas et al., 2010). A key to achieve this is taking the correct decision on optimal treatment and where to transport the patient; severely injured should be taken to a trauma center, having the expertise to treat major injury (Haas et al., 2010; MacKenzie et al., 2006). The prehospital personnel's, i.e. typically the crew in road and air ambulances, most important decision support is the triage protocol. It facilitates identifying patients with severe injury, while using health care resources efficiently by recognizing patients not likely to be in need of urgent and specialized care. In Sweden the Rapid Emergency Trauma and Triage System (RETTS) (Widgren and Jourak, 2011) is the most widespread protocol.

Developing and maintaining a highly accurate triage protocol requires detailed and updated knowledge about what type of traffic accidents are most dangerous. Statistics about mortality are rather well established but there are few publications studying how injury severity is linked to Mechanisms of Injury (MOI)/Accident Characteristics (AC). MOI is commonly used in trauma care to refer to the cause of injury (Brown et al., 2011). In this paper the broader term AC is used to refer to characteristics of the accident such as type of collision, posted speed limit, characteristics of the vehicles involved and their occupants. The importance of AC lie in their potential to predict occult injuries and thereby reducing undertriage. A retrospective study including data of around a million trauma patients concluded that using physiologic and anatomic criteria alone lead to undertriage, and strongly supported the use of MOI in trauma and triage systems (Brown et al., 2011).

The aim of this study is to develop an On Scene Injury Severity Prediction (OSISP) algorithm using only AC that are feasible to assess at the scene of accident.

The purpose of developing the model is to identify important factors for improving triage protocols used in Sweden and possibly in other countries with similar traffic environments. Furthermore, that knowledge may help to guide future traffic safety efforts. The long-term goal is to incorporate an OSISP algorithm into the triage/decision support system used by the prehospital personnel, to increase triage accuracy and minimize delay to definitive treatment by e.g. bypassing the closest medical facility in favor of a trauma center when appropriate. The system is aimed primarily at ambulance personnel. In Sweden traffic accidents are usually first handled by the public-safety answering point reached at the common emergency telephone number 112, whose operators are in direct contact with dispatch management for ambulance, fire brigade and police. A road ambulance is usually the first or one of the first resources to reach the scene of accident and its crew has the medical responsibility. The system could be implemented on e.g. a tablet computer to make it easy-to-use in the field for ambulance crew.

To gain understanding of how modern triage systems are designed the Guidelines for Field Triage of Injured Patients Recommendations of the National Expert Panel on Field Triage (Sasser et al., 2012) used in US and RETTS (Widgren and Jourak, 2011) in Sweden can be reviewed. In both systems the first step is based on physiological criteria, such as pulse and breathing rate, and the second step is anatomical criteria of identified injuries, e.g. type of fractures. If any of the criteria in the first two steps is fulfilled the patient is given the highest priority level (red in RETTS). Next, in step three, MOI are examined. If any of the MOI inclusion criteria are met the patient is given second highest priority level in RETTS (orange). This differs from the US protocol where patients that meet MOI criteria should be handled as stated in (Sasser et al., 2012): "Transport to a trauma center, which, depending upon the defined trauma system, need not be the highest level trauma center".

Focusing on the MOI that apply for motor vehicle crashes some criteria are shared between both triage systems but differences are also found. Both systems include death in the same car and occupant being ejected from the vehicle. Rollover accident was previously a criteria in the American system but it has been removed in the two last versions of the protocol. RETTS includes rollover as criteria, 
as well as trapped occupant. There are two other criteria in RETTS that apply to car accidents, estimated speed $>60 \mathrm{~km} / \mathrm{h}$ and deployment of airbag. These have no direct equivalent criteria in the US system, which recommends to measure intrusion of the occupant compartment to assess the forces having acted upon the occupant(s). Furthermore, the expert panel recommends to utilize vehicle telemetry data (transmitted from vehicle to dispatch center) consistent with high risk of injury when available (Sasser et al., 2012). In the development of the URGENCY algorithm (Augenstein et al., 2003) for identifying severe crashes and a similar algorithm for the OnStar R) system (Kononen et al., 2011) seat belt use, direction and location of impact, and delta- $\mathrm{V}$, i.e. the total change in velocity, were recognized as important predictors of severe injury that can be recorded by telemetry systems integrated in vehicles (incorporating sensors such as accelerometers and gyroscopes to measure impact forces). However, the use of telemetry systems is currently limited in Sweden and Europe, thus algorithms that can predict risk of severe injury as a function of AC that can be quickly assessed at the scene of accident would be valuable.

\section{Materials and Methods}

\subsection{Data Selection}

The scope of this study is adult car occupants in traffic accidents registered in the Swedish Traffic Accident Data Acquisition (STRADA) database for calendar years 2003 to 2013. STRADA is the Swedish Transport Administrations national information system for traffic accidents occurring on the Swedish road network. It contains information reported by the police, and medical data on injured road users treated at emergency hospitals reporting to STRADA. The police visits the scene of accident and reports the circumstances whereas the hospitals determine the injuries the patient sustains and their degree of severity. By combining data from two sources the STRADA system can provide more comprehensive information on the circumstances and consequences of road traffic accidents. The hospital reports focus on the individual level, i.e. the patient, as opposed to the police reports that focus on the circumstances of the accident, which may involve multiple casualties. The database is contained in a Microsoft Access $\mathrm{R}$ database file. More information about STRADA can be found in (Howard and Linder, 2014).

There are over 600000 injured patients registered in the hospital dataset of STRADA from 2003-2013. In the database every accident has a unique accident number, which is the link between the police reported and hospital reported information. Only accidents containing both reports were used in this study. Thereafter adults of age $\geq 18$ years were selected. The time span considered was from 2003, when the police started to systematically report to STRADA, until and including accidents reported in 2013. Only the entries with no missing data in any of the model variables were selected. After this selection process 29128 casualties injured in 22607 accidents remained.

\subsection{Injury severity assessment and dichotomization}

In order to classify occupants as severely injured or not the Injury Severity Score (ISS) (Baker and O'Neill, 1976) was used. ISS is based on the classification of the severity of each injury according to the Abbreviated Injury Scale (AIS) (AAAM, 2005). Two different thresholds were used to define severe injury: ISS $>15$ and ISS $>8$.

\subsection{Variables included in the model}

The dependent variable is whether the patient is classified as being severely injured or not. Two sets of models were developed for the two different levels of severity, i.e. ISS $>8$ and ISS $>15$, respectively. All variables included in the model are detailed in Table 1. 
Table 1: Definitions and descriptions of the model variables. For each variable level the proportion (frequency) of the casualties having that characteristic is shown. The source of the data is given in the second column as Police Report (PR), Hospital Report (HR) or matched (M) in STRADA, where M means that the information was available in both PR and HR and was congruent. Note that the HR variables Belt Use, Airbag and Type are self-reported by the patient.

\begin{tabular}{|c|c|c|c|}
\hline Variable & Definition/source & Level/Frequency & Description \\
\hline ISS & $\begin{array}{l}\text { Injury Severity } \\
\text { Score }(\mathrm{HR})\end{array}$ & $\begin{array}{l}\text { ISS } \leq 8(95.1 \%) \\
8<\operatorname{ISS}<15(2.9 \%) \\
\text { ISS }>15(2.0 \%)\end{array}$ & $\begin{array}{l}\text { Whether the occupant was severely injured or not (dependent variable). } \\
\text { The thresholds used for the two models were ISS }>8 \text { and ISS }>15 \text {, with } \\
4.9 \% \text { and } 2.0 \% \text { of casualties above the threshold, respectively. }\end{array}$ \\
\hline Belt Use & Belt use (HR) & $\begin{array}{l}\text { Belted }(94.1 \%) \\
\text { Unbelted }(5.9 \%)\end{array}$ & Whether the occupant used seat belt \\
\hline Airbag & $\begin{array}{l}\text { Airbag } \\
\text { Deployment (HR) }\end{array}$ & $\begin{array}{l}\text { Not deployed }(59.6 \%) \\
\text { Deployed }(38.3 \%) \\
\text { No Airbag }(2.1 \%)\end{array}$ & Whether the airbag (if present) was deployed \\
\hline Type & $\begin{array}{l}\text { Type of } \\
\text { accident }(\mathrm{HR})\end{array}$ & $\begin{array}{l}\text { Single }(31 \%) \\
\text { Intersection }(22.8 \%) \\
\text { Longitudinal }(12.7 \%) \\
\text { Rear End }(27 \%) \\
\text { Tram/Train }(0.2 \%) \\
\text { WLA }(3.2 \%) \\
\text { Other }(3.1 \%)\end{array}$ & $\begin{array}{l}\text { Single vehicle collides with stationary object or departs from the road } \\
\text { Collision (head on or side) with another vehicle in an intersection } \\
\text { Collision (head on or side) with another vehicle outside an intersection } \\
\text { Vehicle impacts another vehicle from behind } \\
\text { Collision with a tram or a train } \\
\text { Collision with a wild life animal (WLA), in most cases a moose } \\
\text { Other type of accident }\end{array}$ \\
\hline PSL & $\begin{array}{l}\text { Posted speed } \\
\text { limit }(\mathrm{PR})\end{array}$ & $\begin{array}{l}20 \mathrm{~km} / \mathrm{h}(0.01 \%) \\
30 \mathrm{~km} / \mathrm{h}(1.2 \%) \\
40 \mathrm{~km} / \mathrm{h}(0.8 \%) \\
50 \mathrm{~km} / \mathrm{h}(29.1 \%) \\
60 \mathrm{~km} / \mathrm{h}(1 \%) \\
70 \mathrm{~km} / \mathrm{h}(31 \%) \\
80 \mathrm{~km} / \mathrm{h}(4.3 \%) \\
90 \mathrm{~km} / \mathrm{h}(20.1 \%) \\
100 \mathrm{~km} / \mathrm{h}(2.9 \%) \\
110 \mathrm{~km} / \mathrm{h}(8.8 \%) \\
120 \mathrm{~km} / \mathrm{h}(0.7 \%)\end{array}$ & Maximum speed allowed where the accident occurred \\
\hline Location & $\begin{array}{l}\text { Location of } \\
\text { accident }(\mathrm{PR})\end{array}$ & $\begin{array}{l}\text { Urban }(37.6 \%) \\
\text { Rural }(62.4 \%)\end{array}$ & $\begin{array}{l}\text { Whether accident occurred in urban or rural environment, i.e. inside or } \\
\text { outside a population center. Circumvallation roads defined as urban. }\end{array}$ \\
\hline Position & Seat position $(\mathrm{PR})$ & $\begin{array}{l}\text { Front }(94.5 \%) \\
\text { Back }(4.5 \%)\end{array}$ & Seat position of the injured occupant. Divided into front or back seat. \\
\hline Elderly & $\begin{array}{l}\text { Victim over or under } \\
55 \text { years old }(\mathrm{M})\end{array}$ & $\begin{array}{l}\leq 55(78.4 \%) \\
>55(21.6 \%)\end{array}$ & $\begin{array}{l}\text { Age } 55 \text { years old is used as threshold. The mean age was } 39 \text { years and } 8 \\
\text { months. }\end{array}$ \\
\hline Sex & Sex of occupant (M) & $\begin{array}{l}\text { Male }(53.6 \%) \\
\text { Female }(46.4 \%)\end{array}$ & Male or female occupant \\
\hline Period & Calendar years (HR) & $\begin{array}{l}2003-2006(18.8 \%) \\
2007-2013(81.2 \%)\end{array}$ & $\begin{array}{l}\text { In January } 2007 \text { the injury coding system was changed from AIS-1990 to } \\
\text { AIS-2005, causing a substantial effect towards lower levels of injury. }\end{array}$ \\
\hline
\end{tabular}




\subsection{Data Analysis}

All statistical calculations were performed with IBM SPSS Version 22. Univariate chi-squared tests of association were used to compute $p$-values under the null hypothesis of no association between the different explanatory variables and injury severity. Next multivariate analyses were performed. Logistic regression modeling was used to discriminate between severe and non-severe injury and compute the odds ratio (OR) of severe injury for each explanatory variable. Logistic regression is a maximum-likelihood method that is commonly used in studies of traffic accidents, see e.g. (Harrell, 2001; Schiff et al., 2008; Augenstein et al., 2003; Kononen et al., 2011).

A binary logistic regression model fits the logs odd (logit) by a linear function of the explanatory variables (Equation 1).

$$
\ln \left(\frac{P(Y=1)}{1-P(Y=1)}\right)=\beta_{0}+\sum_{k=1}^{n} \beta_{k} x_{k}
$$

$Y$ is the dependent variable, $Y=0$ for non-severe injury and $Y=1$ for severe injury. $P$ denotes the probability of severe injury. The OR for the $k_{t h}$ variable are $e^{\beta_{k}}$. Results of logistic regression modeling are expressed as adjusted OR, with corresponding 95\% confidence intervals (CI). Because of the overall low proportion of severe injury in this study $(<5 \%)$ OR can be considered reasonable approximations of the relative risk.

The area under the receiver operator characteristic (ROC) curve (AUC) was used to measure the performance of the model. The ROC shows the power in terms of sensitivity and specificity for prediction of severe injury for different cutoff values of $P(Y=1)$ (Equation 1). The cutoff value determines the trade-off between sensitivity and specificity; increasing the sensitivity (identifying more patients with severe injury) is at the cost of decreasing the specificity (more false positives, i.e. more patients that are not severely injured are predicted to have severe injury). Use of the OSISP algorithm in the field will require finding a suitable value for this cutoff. This is best determined by the management for health care trauma systems and is outside the scope of this study.

A 10-fold cross validation (CV) procedure was performed to estimate the performance of the OSISP algorithm for unseen data, in terms of ROC and AUC. The dataset was divided into ten randomized folds with approximately equal number of casualties. One fold at a time was left out, a model was derived using Equation 1 on data from the remaining nine folds. The model was then validated by classifying the observations in the left out fold. This procedure was repeated for all folds, i.e. performed ten times.

\section{Results}

Two models corresponding to the thresholds ISS $>15$ and ISS $>8$ are presented. For each model, first the univariate analysis is shown, including: 1) Degrees of freedom and $p$-value for each variable; 2) Proportion of severely injured patients associated with each level of each variable; and 3) The influence of age on the probability of sustaining severe injury. Then the logistic regression model is presented showing: 1) Levels of statistical significance ( $p$-values), OR and $95 \%$ CI for each variable; 2) The ROC, AUC and corresponding CI.

\subsection{Model for Severe Injury when $I S S>8$}

In Table 2 it is shown that all variables except Position are statistically significant $(p<0.05)$ for the univariate analyses. Figure 1 shows that age is strongly associated with severe injury.

Table 3 shows the results for the multivariate model. The ROC of the multivariate model is shown in Figure 2 and AUC values are presented in Table 4. It can be seen that the full model and the 10-fold CV estimate have very similar performance.

From the uni- and multivariate analyses Belt Use stands out as the most valuable predictor. It can be observed that longitudinal accidents in a rural environment where the posted speed limit is $70-100 \mathrm{~km} / \mathrm{h}$ 
are the most dangerous, whereas rear-end accidents in an urban environment are the least dangerous. Regarding the patient perspective age $>55$ years has the strongest influence on the probability of sustaining severe injury. Airbag deployment is not strongly associated with injury severity; accidents where the airbag deploy are slightly more dangerous and cars equipped with airbag are safer than cars without.

Table 2: Results for the univariate analysis for ISS $>8 . \mathrm{DF}=$ Degrees of Freedom. The right-most column shows the proportion of severely injured for the specific variable level. Note that for PSL $=20 \mathrm{~km} / \mathrm{h}$ there were only three accidents whereof one produced a severe injury.

\begin{tabular}{|c|c|c|c|c|}
\hline Variable & $\mathrm{DF}$ & $p$-value & Level & ISS $>8$ \\
\hline \multirow[t]{2}{*}{ Belt Use } & 1 & $<10^{-4}$ & Unbelted & $17 \%$ \\
\hline & & & Belted & $4.1 \%$ \\
\hline \multirow{3}{*}{ Airbag } & 1 & $<10^{-4}$ & Not deployed & $3.9 \%$ \\
\hline & & & Deployed & $6.3 \%$ \\
\hline & & & No Airbag & $6.9 \%$ \\
\hline \multirow{7}{*}{ Type } & 6 & $<10^{-4}$ & Single & $6.2 \%$ \\
\hline & & & Intersection & $3.5 \%$ \\
\hline & & & Longitudinal & $12 \%$ \\
\hline & & & Rear End & $1.1 \%$ \\
\hline & & & Tram/Train & $6.1 \%$ \\
\hline & & & Wild Animal & $5.3 \%$ \\
\hline & & & Other & $5.0 \%$ \\
\hline \multirow[t]{11}{*}{ PSL } & 10 & $<10^{-4}$ & $20 \mathrm{~km} / \mathrm{h}$ & $33 \%$ \\
\hline & & & $30 \mathrm{~km} / \mathrm{h}$ & $2.3 \%$ \\
\hline & & & $40 \mathrm{~km} / \mathrm{h}$ & $2.0 \%$ \\
\hline & & & $50 \mathrm{~km} / \mathrm{h}$ & $2.8 \%$ \\
\hline & & & $60 \mathrm{~km} / \mathrm{h}$ & $2.0 \%$ \\
\hline & & & $70 \mathrm{~km} / \mathrm{h}$ & $5.3 \%$ \\
\hline & & & $80 \mathrm{~km} / \mathrm{h}$ & $6.1 \%$ \\
\hline & & & $90 \mathrm{~km} / \mathrm{h}$ & $7.7 \%$ \\
\hline & & & $100 \mathrm{~km} / \mathrm{h}$ & $5.1 \%$ \\
\hline & & & $110 \mathrm{~km} / \mathrm{h}$ & $4.1 \%$ \\
\hline & & & $120 \mathrm{~km} / \mathrm{h}$ & $3.7 \%$ \\
\hline \multirow{2}{*}{ Location } & 1 & $<10^{-4}$ & Urban & $2.7 \%$ \\
\hline & & & Rural & $6.1 \%$ \\
\hline \multirow[t]{2}{*}{ Position } & 1 & 0.13 & Front seat & $4.8 \%$ \\
\hline & & & Back seat & $5.7 \%$ \\
\hline \multirow[t]{2}{*}{ Elderly } & 1 & $<10^{-4}$ & $\leq 55$ & $3.7 \%$ \\
\hline & & & $>55$ & $9.1 \%$ \\
\hline \multirow[t]{2}{*}{ Sex } & 1 & $<10^{-4}$ & Male & $3.7 \%$ \\
\hline & & & Female & $5.9 \%$ \\
\hline \multirow[t]{2}{*}{ Period } & 1 & $<10^{-4}$ & 2003-2006 & $7.3 \%$ \\
\hline & & & $2007-2013$ & $4.3 \%$ \\
\hline
\end{tabular}

\subsection{Model for Severe Injury when ISS $>15$}

In Table 5 it is shown that all variables are statistically significant for the univariate analyses $(p<0.05)$. Figure 1 shows how the risk of severe injury depends on age. Table 6 shows the results for 


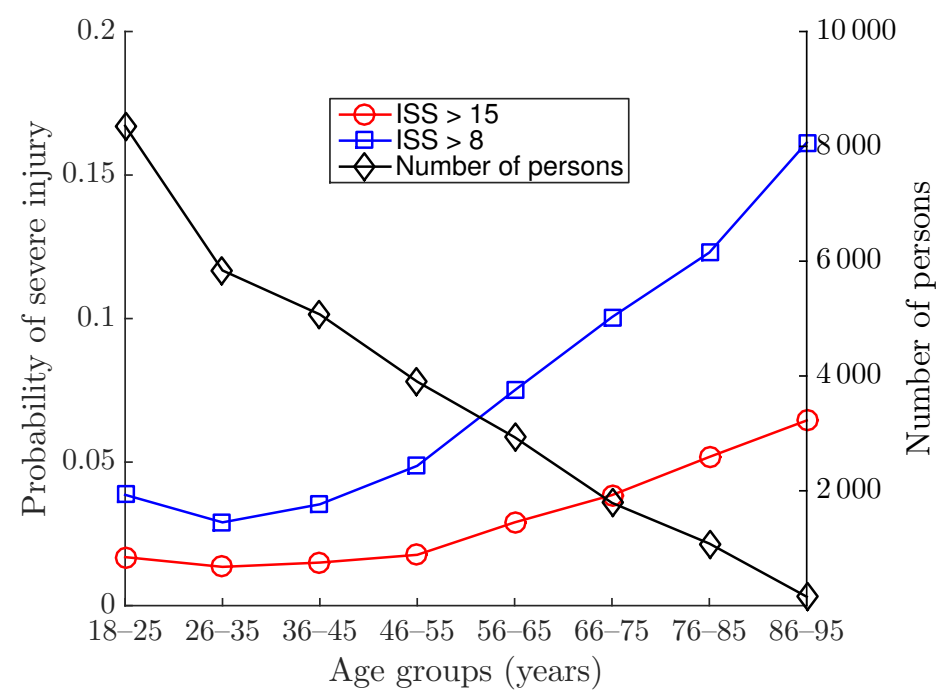

Figure 1: The probability of sustaining severe injury for different age groups (left y-axis) and the person count for each age group (right y-axis).

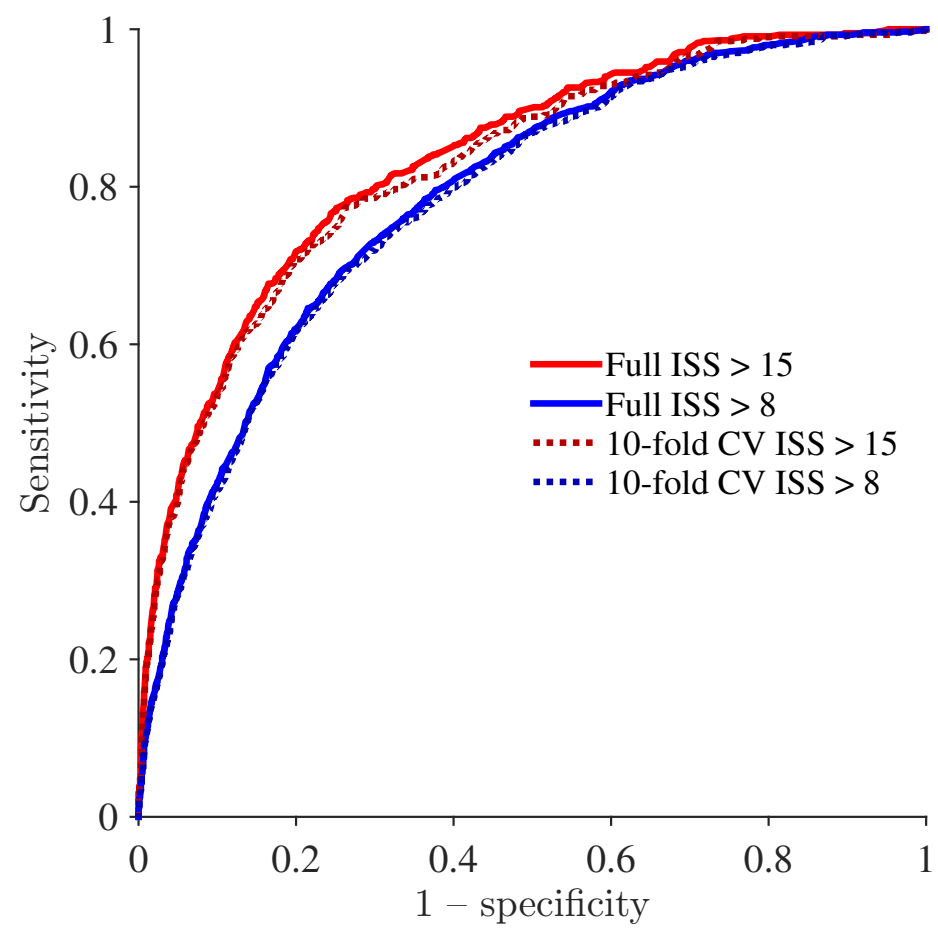

Figure 2: ROC curves for the full models with thresholds ISS $>8$ and ISS $>15$ and corresponding curves for 10-fold cross-validation $(\mathrm{CV})$. 
Table 3: Results for the multivariate analysis for ISS $>8 . \mathrm{DF}=$ Degrees of Freedom. The variable level used as reference is shown as the last level given (for predictors with two levels) or within parenthesis (for predictors with more than two levels).

\begin{tabular}{lrrll}
\hline Variable & $\beta$ & DF & $p$-value & OR $\left(e^{\beta}\right)[95 \% \mathrm{CI}]$ \\
\hline Belted/Unbelted & -1.7 & 1 & $<10^{-4}$ & $0.18[0.15,0.21]$ \\
Airbag & & & & \\
(Not deployed) & & 2 & $<10^{-4}$ & \\
Deployed & 0.32 & 1 & $<10^{-4}$ & $1.4[1.2,1.6]$ \\
No Airbag & 0.47 & 1 & 0.007 & $1.6[1.1,2.2]$ \\
Type & & & & \\
(Rear end) & & 6 & $<10^{-4}$ & \\
Intersection & 1.1 & 1 & $<10^{-4}$ & $2.9[2.3,3.8]$ \\
Longitudinal & 2.2 & 1 & $<10^{-4}$ & $8.8[6.9,11]$ \\
Other & 1.3 & 1 & $<10^{-4}$ & $3.5[2.4,5.1]$ \\
Single & 1.4 & 1 & $<10^{-4}$ & $4.3[3.4,5.4]$ \\
Tram/Train & 2.0 & 1 & 0.001 & $7.4[2.2,25]$ \\
WLA & 1.2 & 1 & $<10^{-4}$ & $3.4[2.4,4.9]$ \\
PSL (30 km/h) & & 10 & $<10^{-4}$ & \\
$20 \mathrm{~km} / \mathrm{h}$ & 2.6 & 1 & 0.041 & $14[1.1,170]$ \\
$40 \mathrm{~km} / \mathrm{h}$ & 0.40 & 1 & 0.50 & $1.5[0.47,4.7]$ \\
$50 \mathrm{~km} / \mathrm{h}$ & 0.36 & 1 & 0.33 & $1.4[0.69,3.0]$ \\
$60 \mathrm{~km} / \mathrm{h}$ & 0.064 & 1 & 0.91 & $1.1[0.35,3.2]$ \\
$70 \mathrm{~km} / \mathrm{h}$ & 0.65 & 1 & 0.081 & $1.9[0.92,4.0]$ \\
$80 \mathrm{~km} / \mathrm{h}$ & 0.73 & 1 & 0.062 & $2.1[0.96,4.5]$ \\
$90 \mathrm{~km} / \mathrm{h}$ & 0.88 & 1 & 0.019 & $2.4[1.2,5.0]$ \\
$100 \mathrm{~km} / \mathrm{h}$ & 0.95 & 1 & 0.019 & $2.6[1.2,5.7]$ \\
$110 \mathrm{~km} / \mathrm{h}$ & 0.61 & 1 & 0.12 & $1.8[0.86,3.9]$ \\
$120 \mathrm{~km} / \mathrm{h}$ & 0.59 & 1 & 0.28 & $1.8[0.62,5.2]$ \\
Rural/Urban & 0.48 & 1 & $<10^{-4}$ & $1.6[1.4,1.9]$ \\
Back/Front & 0.14 & 1 & 0.27 & $1.2[0.89,1.5]$ \\
$>55 / \leq 55$ & 1.0 & 1 & $<10^{-4}$ & $2.7[2.4,3.1]$ \\
Female/Male & 0.31 & 1 & $<10^{-4}$ & $1.4[1.2,1.5]$ \\
$2007-2013 /$ & & & & \\
$2003-2007$ & -0.48 & 1 & $<10^{-4}$ & $0.62[0.54,0.71]$ \\
Constant & -4.0 & 1 & $<10^{-4}$ & 0.018 \\
\hline
\end{tabular}

Table 4: AUC values for multivariate analyses.

\begin{tabular}{lc}
\hline Model & AUC $[95 \% \mathrm{CI}]$ \\
\hline ISS $>8$ & $0.79[0.78,0.80]$ \\
ISS $>8,10$-fold CV & $0.78[0.77,0.79]$ \\
ISS $>15$ & $0.84[0.82,0.85]$ \\
ISS $>15,10$-fold CV & $0.83[0.81,0.84]$ \\
\hline
\end{tabular}


the multivariate model. In general the observations for ISS $>8$ (Table 2 and 3) also apply for ISS $>15$ (Table 5 and 6 ). This means that the uni- and multivariate analyses are consistent across both ISS thresholds.

The ROC of the model is shown in Figure 2. AUC values are presented in Table 4. Again the performance of the full model and 10-fold CV estimate are very similar. The ISS $>15$ model performs better than the ISS $>8$ model.

Table 5: Results for the univariate analysis for ISS $>15$. DF $=$ Degrees of Freedom. The right-most column shows the proportion of severely injured for the specific variable level.

\begin{tabular}{|c|c|c|c|c|}
\hline Variable & DF & $p$-value & Level & $\mathrm{ISS}>15$ \\
\hline \multirow[t]{2}{*}{ Belt Use } & 1 & $<10^{-4}$ & Unbelted & $10 \%$ \\
\hline & & & Belted & $1.5 \%$ \\
\hline \multirow[t]{3}{*}{ Airbag } & 1 & 0.0030 & Not deployed & $1.8 \%$ \\
\hline & & & Deployed & $2.3 \%$ \\
\hline & & & No Airbag & $2.6 \%$ \\
\hline \multirow[t]{7}{*}{ Type } & 6 & $<10^{-4}$ & Single & $2.4 \%$ \\
\hline & & & Intersection & $1.2 \%$ \\
\hline & & & Longitudinal & $6.1 \%$ \\
\hline & & & Rear End & $0.3 \%$ \\
\hline & & & Tram/Train & $2.0 \%$ \\
\hline & & & Wild Animal & $2.0 \%$ \\
\hline & & & Other & $1.9 \%$ \\
\hline \multirow[t]{11}{*}{ PSL } & 10 & $<10^{-4}$ & $20 \mathrm{~km} / \mathrm{h}$ & $0.0 \%$ \\
\hline & & & $30 \mathrm{~km} / \mathrm{h}$ & $0.6 \%$ \\
\hline & & & $40 \mathrm{~km} / \mathrm{h}$ & $0.8 \%$ \\
\hline & & & $50 \mathrm{~km} / \mathrm{h}$ & $0.9 \%$ \\
\hline & & & $60 \mathrm{~km} / \mathrm{h}$ & $1.0 \%$ \\
\hline & & & $70 \mathrm{~km} / \mathrm{h}$ & $2.1 \%$ \\
\hline & & & $80 \mathrm{~km} / \mathrm{h}$ & $2.5 \%$ \\
\hline & & & $90 \mathrm{~km} / \mathrm{h}$ & $4.1 \%$ \\
\hline & & & $100 \mathrm{~km} / \mathrm{h}$ & $1.1 \%$ \\
\hline & & & $110 \mathrm{~km} / \mathrm{h}$ & $0.9 \%$ \\
\hline & & & $120 \mathrm{~km} / \mathrm{h}$ & $1.1 \%$ \\
\hline \multirow[t]{2}{*}{ Location } & 1 & $<10^{-4}$ & Urban & $1.0 \%$ \\
\hline & & & Rural & $2.6 \%$ \\
\hline \multirow[t]{2}{*}{ Position } & 1 & 0.013 & Front & $2.0 \%$ \\
\hline & & & Back & $2.9 \%$ \\
\hline \multirow[t]{2}{*}{ Elderly } & 1 & $<10^{-4}$ & Under 55 & $1.6 \%$ \\
\hline & & & Over 55 & $3.6 \%$ \\
\hline \multirow[t]{2}{*}{ Sex } & 1 & $<10^{-4}$ & Male & $1.4 \%$ \\
\hline & & & Female & $2.5 \%$ \\
\hline \multirow[t]{2}{*}{ Period } & 1 & $<10^{-4}$ & 2003-2006 & $4.1 \%$ \\
\hline & & & $2007-2013$ & $1.5 \%$ \\
\hline
\end{tabular}


Table 6: Results for the multivariate analysis for ISS $>15 . \mathrm{DF}=$ Degrees of Freedom. The variable level used as reference is shown as the last level given (for predictors with two levels) or within parenthesis (for predictors with more than two levels).

\begin{tabular}{lrrll}
\hline Variable & $\beta$ & DF & $p$-value & OR $\left(e^{\beta}\right)[95 \% \mathrm{CI}]$ \\
\hline Belted/Unbelted & -2.1 & 1 & $<10^{-4}$ & $0.13[0.10,0.16]$ \\
Airbag & & & & \\
(Not deployed) & & 2 & 0.33 & \\
Deployed & 0.10 & 1 & 0.32 & $1.10[0.91,1.3]$ \\
No Airbag & 0.34 & 1 & 0.21 & $1.4[0.82,2.4]$ \\
Type & & & & \\
(Rear end) & & 6 & $<10^{-4}$ & \\
Intersection & 1.2 & 1 & $<10^{-4}$ & $3.4[2.2,5.5]$ \\
Longitudinal & 2.7 & 1 & $<10^{-4}$ & $15[9.5,23]$ \\
Other & 1.5 & 1 & $<10^{-4}$ & $4.4[2.4,8.4]$ \\
Single & 1.6 & 1 & $<10^{-4}$ & $5.1[3.3,7.9]$ \\
Tram/Train & 2.3 & 1 & .029 & $9.7[1.3,75]$ \\
WLA & 1.3 & 1 & $<10^{-4}$ & $3.8[2.0,7.1]$ \\
PSL $(30 \mathrm{~km} / \mathrm{h})$ & & 10 & $<10^{-4}$ & \\
$20 \mathrm{~km} / \mathrm{h}$ & -16 & 1 & 1.0 & $0[0,-]$ \\
$40 \mathrm{~km} / \mathrm{h}$ & 1.1 & 1 & 0.29 & $2.9[0.40,21]$ \\
$50 \mathrm{~km} / \mathrm{h}$ & 0.60 & 1 & 0.41 & $1.8[0.44,7.6]$ \\
$60 \mathrm{~km} / \mathrm{h}$ & 0.87 & 1 & 0.35 & $2.4[0.38,15]$ \\
$70 \mathrm{~km} / \mathrm{h}$ & 1.0 & 1 & 0.16 & $2.7[0.66,11]$ \\
$80 \mathrm{~km} / \mathrm{h}$ & 1.2 & 1 & 0.010 & $3.5[0.80,15]$ \\
$90 \mathrm{~km} / \mathrm{h}$ & 1.5 & 1 & 0.041 & $4.4[1.1,18]$ \\
$100 \mathrm{~km} / \mathrm{h}$ & 0.85 & 1 & 0.29 & $2.3[0.49,11]$ \\
$110 \mathrm{~km} / \mathrm{h}$ & 0.40 & 1 & 0.60 & $1.5[0.34,6.5]$ \\
$120 \mathrm{~km} / \mathrm{h}$ & 0.86 & 1 & 0.40 & $2.4[0.32,17]$ \\
Rural/Urban & 0.58 & 1 & $<10^{-4}$ & $1.8[1.4,2.3]$ \\
Back/Front & 0.25 & 1 & 0.18 & $1.3[0.90,1.8]$ \\
$>55 / \leq 55$ & 0.92 & 1 & $<10^{-4}$ & $2.5[2.1,3.0]$ \\
Female/Male & 0.33 & 1 & $<10^{-4}$ & $1.4[1.2,1.7]$ \\
$2007-2013 /$ & -0.87 & 1 & $<10^{-4}$ & $0.42[0.35,0.51]$ \\
$2003-2007$ & & & & \\
Constant & -5.1 & 1 & $<10^{-4}$ & 0.006 \\
\hline
\end{tabular}




\section{Discussion}

\subsection{Statement of findings}

The main finding is that, when the partition between severe and non-severe injury is ISS $>15$, the model developed in this study is a good predictor with an AUC $=0.83$ (Table 4 and Figure 2). For ISS $>8$ as threshold the predictive power of the model is slightly lower, AUC $=0.78$. To exemplify, if a $90 \%$ sensitivity is required the specificity would be $43 \%$ for ISS $>8$ and $47 \%$ for ISS $>15$ (Figure 2 ).

The CV estimates indicates that the full models will perform well on unseen data (Table 4 and Figure 2). However, a prospective study evaluating the model performance for traffic accidents from 2014 and onward is needed to confirm the good performance in practice.

To put these findings into perspective, Harrell (Harrell, 2001) stated that a model with an AUC $>0.80$ has a good discriminating capability. The AUC reported for this study is similar to the one reported in (Kononen et al., 2011). That model was based on vehicle telemetry data, and the dependent variable was vehicles with at least one severely injured occupant. The variables used by (Kononen et al., 2011) could possibly be assessed already at dispatch of rescue and medical personnel, which is the purpose of that algorithm, while our model is thought to be used at the scene of the accident. Our findings indicate that an OSISP algorithm with good predictive power can be achieved without access to telemetry data. Furthermore, the sensitivities and specificities for our model are much higher than the ones reported in (Rehn et al., 2011) for a trauma triage protocol in a Norwegian center. This indicates that the OSISP models developed in this study could be valuable to use in conjunction with established triage protocols such as RETTS.

Regarding explanatory variables belt use is the most strongly related variable to probability of severe injury. Type and location of accident, elderly occupant and posted speed limits are also important predictors. Airbag deployment, sex and seat position (front or back) are weakly related to injury severity. It appears to be slightly safer to sit in the front seat than in the back seat. However; the reason is probably that frequency of unbelted in the back seat was much higher than in the front seat, i.e. $16 \%$ against $5.4 \%$.

A very high risk of sustaining severe injury was observed for longitudinal accidents (head on or side outside an intersection), which is normally caused by a high delta-V in these crashes. Single vehicle accidents show a high risk, whereas rear-end collisions have a low risk, as do intersection accidents. If the accident is located in an urban environment it has lower associated risk compared to a rural environment. Roads with posted speed limit of $70-100 \mathrm{~km} / \mathrm{h}$ have a high associated risk.

Figure 1 show that frequency of severe injury does not change much with age up to approximately 55 years old, but from this age the injury level aggravate with increasing age. This result supports the recommended age partition at 55 years old recommended by the expert panel on field triage (Sasser et al., 2012). It is consistent with the strong association between age and severe injury reported in several studies (MacKenzie et al., 2006; Champion et al., 2005, 1990; Tavris et al., 2001).

The results show that females have a higher probability of sustaining severe injury than males, which is consistent with other studies (Schiff et al., 2008; Rowe et al., 2004; Sampalis et al., 2009). This association may be higher than found in this study due to the tendency of young males to be involved in more severe accidents (Tavris et al., 2001; Kockelman and Kweon, 2002), which could not be adjusted for. This tendency is implied in Figure 1 showing a slightly higher probability of severe injury among subjects 18-25 years old than subjects 26-35 years old.

\subsection{Implications of the findings}

The main implication is the possibility of developing a prehospital tool for field triage of traffic accident victims based on the implementation of the models presented in this paper.

For guidance of where to focus efforts for increasing traffic safety the findings suggest that further education/information campaigns to increase the use of seat belt would be useful. Although belt use 
is very high in Sweden, 97-98\% in year 2013 (Larsson et al., 2014), further improvement would be beneficial. Other very important factors were the type of accident and where it occurred. Longitudinal accidents (head on or side outside an intersection) on rural roads with posted speed limit of $70-100 \mathrm{~km} / \mathrm{h}$ show the highest risk. This finding reinforces the importance of improving safety of rural roads where the risk for frontal crashes is high, i.e. roads with adjacent lanes with traffic flow going in opposite directions not separated by barrier.

The weak association of severe injury to airbag deployment does not mean it is an ineffective crash safety measurement, because crashes for which airbags deploy are in general more violent. However, it casts doubts about using airbag deployment as triage criteria, which is currently included in RETTS. Our results indicate that implementing belt use as MOI criteria, which is currently not included in RETTS, could increase triage accuracy. Our findings need to be confirmed in a prospective study.

\subsection{Limitations of the study}

Most accidents in STRADA were discarded due to missing data in one or more variables. Nevertheless, the statistical power of the analysis was high. It is unclear whether multiple elimination of data is advantageous over data imputation (Kononen et al., 2011). However, since the number of samples and resulting statistical power was high we chose to eliminate data.

While there are many ways to dichotomize between severe and non-severe injury the US National Expert Panel recommended ISS > 15 as partition threshold (Sasser et al., 2012). In addition a substantial majority of data registries (Lossius et al., 2001; MacLeod et al., 2003) use that threshold for defining very severe injury. ISS > 15 was first described by (Boyd et al., 1987) as being predictive of $10 \%$ mortality and it is widely accepted as corresponding to the highest priority level in an Emergency Department (ED) (Palmer, 2007). For comparison we chose to use an alternative definition of severe injury, ISS $>8$, which is the lowest threshold for severe injury that has been suggested (Howard and Linder, 2014). Furthermore, it means that a patient with AIS of level 3 is classified as severe, and is thus similar to maximum AIS $\geq 3$ that is commonly used in traffic accident analysis. The classification performance was similar for both ISS $>8$ and ISS $>15$, which indicate that the model is robust to variations of severe injury definition. The use of more than one threshold could be useful for integrating OSISP with some triage protocols. For example, RETTS uses two levels of higher priority (orange and red). Fulfillment of any MOI criteria will assign the patient to the second highest priority (orange), if no physiological/anatomical criteria for highest level (red) already are fulfilled. A possible way to integrate OSISP with RETTS would be to assign patients who are flagged by OSISP as ISS $>8$ to the orange level, and patients with predicted ISS $>15$ to the red level.

AIS/ISS classification is available for essentially all injuries registered in STRADA. In 2007 injury coding in STRADA was upgraded to AIS-2005, which brought an apparent general trend to lower injury scores (Howard and Linder, 2014; Barnes et al., 2009), e.g. some injuries previously coded as 3 were changed to 2. This had a substantial effect on STRADA (Fredlund, 2009). We introduced the variable Period to adjust for this effect. From 2003-2006 $7.3 \%$ of the patients sustained ISS $>8$ and $4.3 \%$ had ISS $>15$ (Table 2 and Table 5). For the period 2007-2013 4.1\% of the subjects sustained ISS $>8$ and $1.5 \%$ had ISS $>15$. This difference may be attributed to the change from AIS 1990 to AIS 2005, and potentially to safer traffic environments and vehicles.

Information about the car such as model year and weight were not considered due to the perceived difficulty of specifying these variables at the scene of accident. In future OSISP algorithms vehicle telemetry data could provide such information. Death in the same car was not included in the model because it is already a well established predictor of occult injuries and because there were very few fatal crashes with more than one occupant in the vehicle.

STRADA does not provide detailed information about some presumably important variables, most importantly delta-V. Information about the crash such as the angle of impact and compartment intrusion could have improved model accuracy. Furthermore, the variables belt use, airbag and type of accident 
from the hospital report in STRADA are self-reported by the patient. This may lead to lower accuracy of the database, which may impair validity of the OSISP models derived in this study. However, it is expected that higher accuracy of reported data would lead to better performance of the OSISP algorithms. This indicates that the method has a high potential to be used as a complementary tool for field triage.

\section{Conclusion}

An OSISP algorithm was developed for predicting the probability of a car occupant being severely injured following a crash using only AC that can be rapidly assessed at the scene of accident by prehospital personnel. The algorithm showed a good capability of differentiating severe and non-severe injury and could be utilized to improve triage accuracy in conjunction with current triage protocols.

\section{Acknowledgements}

This work has been carried out at SAFER - Vehicle and Traffic Safety Centre at Chalmers, Sweden.

\section{References}

AAAM, 2005. Abbreviated Injury Scale. Association for the advancement of automotive medicine, Barrington, IL, USA .

Augenstein, J., Perdeck, E., Stratton, J., Digges, K., Bahouth, G., 2003. Characteristics of crashes that increase the risk of serious injuries, in: Annu Proc Assoc Adv Automot Med, pp. 561-576.

Baker, S.P., O’Neill, B., 1976. The Injury Severity Score. The Journal of Trauma: Injury, Infection, and Critical Care 16, 882-885.

Barnes, J., Hassan, A., Cuerden, R., Cookson, R., Banbury, J., Kohlhofer, J., 2009. Comparison of injury severity between AIS 2005 and AIS 1990 in a large injury database, in: Annals of Advances in Automotive Medicine, Vol. 53. 53rd Annual Scientific Conference, Baltimore, Maryland, USA, 5th-7th October, pp. 83-89.

Boyd, C.R., Tolson, M.A., Copes, W.S., 1987. Evaluating Trauma Care. The Journal of Trauma: Injury, Infection, and Critical Care 27, 370-378.

Brown, J.B., Stassen, N.A., Bankey, P.E., Sangosanya, A.T., Cheng, J.D., Gestring, M.L., 2011. Mechanism of Injury and Special Consideration Criteria Still Matter: An Evaluation of the National Trauma Triage Protocol. Journal of Trauma-Injury Infection and Critical Care 70, 38-45.

Champion, H., Augenstein, J., Blatt, A., Cushing, B., Digges, K., Flanigan, M., Hunt, R., Lombardo, L., Siegel, J., 2005. New tools to reduce deaths and disabilities by improving emergency care: URGENCY software, occult injury warnings, and air medical services database, in: Proceedings of the 19th International Technical Conference on Enhanced Safety of Vehicles (NHTSA sponsored), Washington, DC.

Champion, H.R., Copes, W.S., Sacco, W.J., Lawnick, M.M., Keast, S.L., Frey, C.F., 1990. The Major Trauma Outcome Study. The Journal of Trauma: Injury, Infection, and Critical Care 30, 1356-1365.

Fredlund, T., 2009. Färre allvarligt skadade i vägtrafiken - Effekten av förändrad skadeklassificering. Transportstyrelsen TSV 2009-14721, 1-29. 
Haas, B., Gomez, D., Zagorski, B., Stukel, T.A., Rubenfeld, G.D., Nathens, A.B., 2010. Survival of the Fittest: The Hidden Cost of Undertriage of Major Trauma. Journal of the American College of Surgeons 211, 804-811. doi:DOI 10.1016/j.jamcollsurg.2010.08.014.

Harrell, F.E., 2001. Regression modeling strategies, with applications to linear models, survival analysis and logistic regression. Springer Science and Business Media, Inc., New York.

Howard, C., Linder, A., 2014. Review of Swedish experiences concerning analysis of people injured in traffic accidents. Swedish National Road and Transport Research Institute (VTI) VTI notat 7A-2014, $1-34$.

Kockelman, K.M., Kweon, Y.J., 2002. Driver injury severity: an application of ordered probit models. Accident Analysis \& Prevention 34, 313-321.

Kononen, D.W., Flannagan, C.A.C., Wang, S.C., 2011. Identification and validation of a logistic regression model for predicting serious injuries associated with motor vehicle crashes. Accident Analysis \& Prevention 43, 112-122.

Larsson, J., Henriksson, P., Yahya, M.R., 2014. The use of seat belts in Sweden 2013. Swedish National Road and Transport Research Institute (VTI) VTI notat 10-2014, 1-41.

Lossius, H.M., Langhelle, A., Eldar, S., Pillgram-Larsen, J., Lossius, T.A., Laake, P., Steen, P.A., et al., 2001. Reporting data following major trauma and analysing factors associated with outcome using the new Utstein style recommendations. Resuscitation 50, 263-272.

MacKenzie, E., Rivara, F., Jurkovich, G., Nathens, A., Frey, K., Egleston, B., Salkever, D., Scharfstein, D., 2006. A national evaluation of the effect of trauma-center care on mortality. New England Journal of Medicine 354, 366-378.

MacLeod, J.B.A., Kobusingye, O., Frost, C., Lett, R., Kirya, F., Shulman, C., 2003. A Comparison of the Kampala Trauma Score (KTS) with the Revised Trauma Score (RTS), Injury Severity Score (ISS) and the TRISS Method in a Ugandan Trauma Registry. European Journal of Trauma 29, 392-398.

Murad, M.K., Larsen, S., Husum, H., 2012. Prehospital trauma care reduces mortality. Ten-year results from a time-cohort and trauma audit study in Iraq. Scand J Trauma Resusc Emerg Med 20, 13.

Palmer, C., 2007. Major Trauma and the Injury Severity Score - Where Should We Set the Bar?, in: Annual Proceedings/Association for the Advancement of Automotive Medicine, p. 13.

Rehn, M., Perel, P., Blackhall, K., Lossius, H., 2011. Prognostic models for the early care of trauma patients: a systematic review. Scand J Trauma Resusc Emerg Med 19, 17.

Rowe, S.A., Sochor, M.S., Staples, K.S., Wahl, W.L., Wang, S.C., 2004. Pelvic ring fractures: Implications of vehicle design, crash type, and occupant characteristics. Surgery 136, 842-847.

Sampalis, J.S., Nathanson, R., Vaillancourt, J., Nikolis, A., Liberman, M., Angelopoulos, J., Krassakopoulos, N., Longo, N., Psaradellis, E., 2009. Assessment of Mortality in Older Trauma Patients Sustaining Injuries from Falls or Motor Vehicle Collisions Treated in Regional Level I Trauma Centers. Annals of Surgery 249, 488-495.

Sasser, S., Hunt, R., Faul, M., Sugerman, D., Pearson, W., Theresa, D., Wald, M., Jurkovich, G., Newgard, C., Lerner, E., Cooper, A., Wang, S., Henry, M., Salomone, J., Galli, R., 2012. Guidelines for Field Triage of Injured Patients - Recommendations of the National Expert Panel on Field Triage, 2011. MMWR 61, 1-20. 
Saxton, B., 2013. Vägtrafikskador 2012. Trafikanalys 2013:9, 1-116.

Schiff, M.A., Tencer, A.F., Mack, C.D., 2008. Risk factors for pelvic fractures in lateral impact motor vehicle crashes. Accident Analysis \& Prevention 40, 387-391.

Tavris, D.R., Kuhn, E.M., Layde, P.M., 2001. Age and gender patterns in motor vehicle crash injuries: importance of type of crash and occupant role. Accident Analysis \& Prevention 33, 167-172.

Widgren, B.R., Jourak, M., 2011. Medical Emergency Triage and Treatment System (METTS): A New Protocol in Primary Triage and Secondary Priority Decision in Emergency Medicine. Journal of Emergency Medicine 40, 623-628. 